Archives

2| 1988

Varia

\title{
Propos en guise d'introduction
}

\section{Marcel Roncayolo}

\section{(apenEdition \\ Journals}

\section{Édition électronique}

URL : http://journals.openedition.org/ccrh/2953

DOI : $10.4000 /$ ccrh.2953

ISSN : $1760-7906$

\section{Éditeur}

Centre de recherches historiques - EHESS

\section{Édition imprimée}

Date de publication : 15 avril 1988

ISSN : 0990-9141

\section{Référence électronique}

Marcel Roncayolo, "Propos en guise d'introduction », Les Cahiers du Centre de Recherches Historiques [En ligne], 2 I 1988, mis en ligne le 13 avril 2009, consulté le 01 mai 2019. URL : http:// journals.openedition.org/ccrh/2953; DOI : 10.4000/ccrh.2953

Ce document a été généré automatiquement le 1 mai 2019.

Article L.111-1 du Code de la propriété intellectuelle. 


\title{
Propos en guise d'introduction
}

\author{
Marcel Roncayolo
}

1 Je voudrais retenir des rencontres de septembre 1987 entre historiens hongrois et français sur l'urbanisation, non pas tel apport particulier, mais des propositions qui me semblent relancer les débats de l'histoire urbaine ou en suggérer d'autres. Postface, plus que présentation : elle n'engage que des réactions personnelles. Ce qui manque peut-être à l'histoire comparée, menée le plus souvent par saccades, c'est d'être construite. Ces quelques lignes ne sont qu'une invite à le faire.

D'abord peut-on parler pour l'ensemble de l'Europe des villes de l'âge industriel ? Il n'est pas question de revenir sur l'opposition classique entre Europe verte et Europe industrielle, que les manuels enseignaient au début de ce siècle; les contrastes d'économie, de structure sociale, de dépendance sont connus. Simplement la rencontre conduit à plus de nuances (survivances ou non de pratiques féodales, place de la noblesse, composition et importance des "bourgeoisies nationales»). Mais au delà? A l'Ouest, le mouvement moderne d'urbanisation ne s'identifie pas mécaniquement avec le phénomène d'industrialisation, du moins dans ses manifestations les plus directes: le travail industriel. En Europe orientale, dans ces économies restées en majorité terriennes, agraires, les villes en expansion, celles qui parviennent à fixer le commerce, les institutions culturelles, le cas échéant le pouvoir et les germes de l'industrie moderne, ne vivent pas à l'abri des contagions plus immédiates de l'industrialisme, du chemin de fer aux tramways, de l'éclairage au gaz à l'électricité, de l'urbanisme aux grands travaux «hygiénistes». Dans une grande mesure, c'est par la gestion de la ville, les services urbains, l'infrastructure que les valeurs industrielles et souvent les grandes entreprises capitalistes s'installent. L'urbanisation, quantitative et qualitative, loin d'être le reflet passif du système de production, parait solliciter la transformation de l'économie. On comprend mieux comment, fort souvent, l'affirmation des capitales semble coïncider avec l'industrie moderne, et c'est le cas en Hongrie.

3 Capitales? Les villes ou mieux l'urbanisation moderne obéissent en Europe orientale à un autre souffle, distinct de l'économie. Sous des formes variées qui tiennent à la situation, au degré de souveraineté, au peuplement, aux discours politiques, l'Europe verte couvre au XIX ${ }^{e}$ siècle des nations en formation; ou mieux, comme en Hongrie, l'ambition d'un 
État national. L'un des mérites de nos collègues hongrois est de montrer l'alliance qui s'établit entre ville et mouvement national, même dans les pays où la noblesse terrienne participe très activement à celui-ci ou le dirige. La ville acquise, lieu de rencontre de populations et de classes, de groupes ethniques ou religieux, d'information. La ville devient véritable acteur collectif; non sans quelques paradoxes. Peut-on abriter le mouvement national hongrois et servir à la prise de conscience slovaque ou croate? A l'inverse, les minorités allemandes ou juives deviennent assez rapidement les agents les plus actifs de la magyarisation. La ville est ainsi un outil, fonctionnel et symbolique, en vue de l'unité et de l'indépendance. Je garde en mémoire le constat de notre collègue Niederhauser qui évoque cette stratégie urbaine du mouvement national, à propos de Pest, au milieu du siècle passé : «L'un des leaders du mouvement hongrois, le comte Szechenyi, déploie de grands efforts pour faire de Pest le centre économique et culturel de la Hongrie et, par là même, du mouvement hongrois ». Préformation d'une capitale ? Faut-il considérer les mouvements nationaux, y compris dans leur histoire évènementielle comme un facteur d'urbanisation et de sélection urbaine? Pourquoi pas? Le modèle qui privilégie la capitale unique, centralisant ensuite les initiatives de l'état comme celles du capital, est puissant, toujours vivace. Il ne se cantonne pas dans l'Europe du XIX ${ }^{\mathrm{e}}$ siècle ou de l'entre-deux-guerres; on le retrouve en Amérique latine, dans les nations africaines, vieilles ou nouvelles, là où les sociétés paysannes le plus souvent l'emportaient. D'autres modèles plus fédératifs ou plus additifs se sont imposés, quand l'armature urbaine était à la fois plus dense, plus divisée, plus conflictuelle, les positions économiques et culturelles des villes riches mieux affirmées. Pensons à l'Italie: les conditions de réalisation de l'unité nationale et de l'État sont en partie liées à la géographie urbaine déjà acquise. Mais elles pèsent aussi sur le destin des villes et, en fin de compte, la manière dont elles assimilent, monopolisent ou contrôlent les formes modernes de l'économie.

Capitale, capitalisme : l'étymologie ne se limite pas à un pur jeu verbal. Guy Burgel ${ }^{1}$ l'avait $^{2}$ déjà démontré pour Athènes. La formation de l'État grec, le choix d'un second destin, national celui-ci, pour l'antique cité, substituent peu à peu aux vieilles dynasties commerçantes (qui ont nourri les premières diasporas), une toute autre forme d'organisation économique et du développement industriel, centrée sur la capitale, mêlant d'ailleurs, d'une manière confuse, les intérêts des affaires et ceux de la politique. Sur le premier point, la centralisation de l'initiative économique, la leçon de Budapest est claire, malgré le téléscopage apparent des périodes. En Hongrie, en effet, les choses vont vite ; en moins d'un demi-siècle, de 1864 à 1910, la capitale accumule les responsabilités politiques, les charges d'une grande agglomération, l'essor de la grande industrie; mais regardons de plus près les enchaînements. L'avance économique et culturelle de Pest se dessine au début de ce siècle : lieu de commerce du blé, Pest fixe une industrie moderne et exportatrice de la minoterie. Comptons avec l'oeuvre de Szechenyi aussi. Dès 1848, la future capitale s'annonce. La création du «dualisme» reste pourtant l'épreuve principale, avec les mesures majeures, administratives et urbanistiques, qui fondent la ville dans son rôle nouveau: intégration de trois cités: Buda, Pest et Obuda; plan d'extension; développement des services urbains; même s'il faut reprendre leur organisation et leur financement quelques vingt ans plus tard. Budapest s'aligne, de ce fait, sur les grandes capitales européennes. Londres ou Paris sont à l'horizon. Vient ensuite le temps des infrastructures, de la circulation ferroviaire (toujours les toiles d'araignée!), de l'équipement portuaire, de l'organisation bancaire. Enfin, la seconde phase d'industrialisation, bien dissociée des activités initiales, faisant place à des formes de concentration financière et technique que la Hongrie ne connaissait pas jusqu'alors, et 
à l'essor de secteurs nouveaux, industries mécaniques puis électriques, par exemple. Je décompose peut-être à l'excès des mouvements qui se chevauchent. Mais la grande industrie vient en bout de course, plus qu'au départ, le développement topographique de Budapest le confirme. Un fait apparemment secondaire illustre cet enchaînement: le glissement professionnel, d'une génération à l'autre, des fis d'employés «urbains » (services publics, transports, chantiers, administration) vers le travail qualifié en usine.

De telles séquences: luttes nationales, affirmation des capitales, industrialisation amènent à s'interroger sur les acteurs sociaux qui conduisent ces changements. La Hongrie, les textes le démontrent aisément, se prête peu à l'application sans réserve de la thèse des bourgeoisies nationales. Le mouvement national est visiblement porté par une noblesse qui s'est dégagée d'une trop lourde tradition féodale, si elle entraîne avec elle une large part des fractions bourgeoises, minorités allemande ou juive en tête. La bourgeoisie se construit, parallèlement à ces changements ; il faut peut-être accorder plus d'importance à la rencontre des élites urbaines ou urbanisées, articulant leurs rôles (au prix des conflits éventuels); une prosopographie scrupuleuse du personnel dirigeant des corps politiques, municipaux et des entreprises aiderait à mieux comprendre ce que l'étude de l'urbanisation de la population juive indique déjà : les Juifs deviennent, semblet-il, le groupe leader de «l'embourgeoisement» de la capitale. Classes en formation plutôt qu'entités d'emblée acquises, voilà les lignes d'une histoire sociale qui ne traiterait pas l'urbain comme un simple cadre géographique. Le riche exposé de notre collègue Lacko me paraît apporter des vues très pertinentes sur la formation de la classe ouvrière, dans le même esprit d'analyse.

Je n'ignore pas que ma sélection de thèmes est injuste, trop partielle ; qu'il faudrait peutêtre s'interroger sur la vieille question du seuil de l'urbain ; on peut sacrifier aisément en Hongrie, comme douteuses et trop chargées de "villages enflés", les fractions élémentaires de l'habitat aggloméré ; jusqu'à 5.000 habitants ou 20.000 habitants. Tracer le portrait urbain de la France du XIXe siècle sans accorder quelque intérêt aux petites villes (selon les critères mêmes, très judicieux, du commentateur Kaleli) serait amputer la vérité sociale et ethnologique de "l'urbanité » française. Pour le reste, aborder d'une manière critique, comparée, les problèmes que nous venons d'évoquer, dans une perspective où l'économie laisse place à d'autres séries explicatives, où les politiques de formation de la Nation et de l'État et les politiques urbaines ont leur place et où, en fin de compte, la ville ne se réduit pas à une simple expression géographique, n'est pas un effort inutile.

\section{NOTES}

1. Guy BURGEL, Athènes : étude de la croissance d'une capitale méditerranéenne. Thèse de l'Université de Paris I, 1974. 


\section{AUTEUR}

MARCEL RONCAYOLO

Marcel RONCAYOLO est professeur à l'Université Paris X et directeur d'études à l'EHESS. 